\title{
Reliable Lifespan Evaluation of a Remote Environment Monitoring System Based on Wireless Sensor Networks and Global System for Mobile Communications
}

\author{
Diego Antolín, Nicolás Medrano, and Belén Calvo \\ Group of Electronic Design (GDE-I3A), University of Zaragoza, 50009 Zaragoza, Spain \\ Correspondence should be addressed to Diego Antolín; dantolin@unizar.es
}

Received 7 September 2015; Revised 19 January 2016; Accepted 21 January 2016

Academic Editor: Fanli Meng

Copyright (c) 2016 Diego Antolín et al. This is an open access article distributed under the Creative Commons Attribution License, which permits unrestricted use, distribution, and reproduction in any medium, provided the original work is properly cited.

\begin{abstract}
The use of wireless sensor networks (WSN) for monitoring physical and chemical variables in large areas allows density and frequency measurements which have been unavailable to date in classical measurement systems. To fully take advantage of this technology in a particular application, besides an accurate design and selection of all the components involved in its operation, it is essential to dispose of reliable lifetime estimation prior to deployment. This paper presents an experimental approach to determine the actual lifetime of such battery-operated systems, making use of a custom WSN architecture, and for different batteries technologies. To render a reliable evaluation, the energy consumption of the sensor nodes under their different operation modes, in correlation with the battery characteristics and the voltage regulation system, is jointly considered. The result is a complete and practical lifetime model, whose appropriate performance has been validated in a real deployment scenario.
\end{abstract}

\section{Introduction}

The fast development of wireless communications systems for the past two decades has favored the emergence of many previously unthinkable applications. An emerging example is that of wireless sensor networks (WSN), composed of a large number of sensor nodes capable of monitoring different physical and chemical magnitudes from the environment in which they are distributed and where the transmission of data is done through a suitable RF module. These systems are mostly based on the Low Rate Wireless Personal Area Network (LR-WPAN) IEEE 802.15.4 communication standard. This standard is designed to be compliant with the requirement of low-power consumption, thus allowing being fed by small batteries, with a lifetime of months or even years.

The limited range of the IEEE 802.15.4 RF modules (usually a few hundred meters) requires the use of techniques of multihop data transmission to cover larger monitoring areas. In multihop transmission, some sensor nodes act as cell stations for data provided by other nodes. Thus, it is possible to achieve greater transmission distances. The information collected by a WSN should be finally transmitted to a central node (usually connected to a PC), which will process and represent the received data in order to get straightforwardly interpretable results.

This work presents the development and characterization of a sensor node platform, validated through real WSN deployment and test. Since nowadays WSN energy efficiency remains a primary challenge for the success of these networks, one key part of the work is the study and analysis of the power consumption with the objective to obtain a simple but reliable experimental lifetime estimation model that prevents a battery network failure.

The paper is structured as follows: Section 2 shortly presents the state of the art about environmental monitoring based on wireless sensor networks. Section 3 provides a brief description of the sensor-router nodes including hardware, software, communication characteristics, and power management techniques. Section 4 addresses the proposed methodology for modeling the battery lifetime of a basic sensor node within the custom WSN platform. Section 5 extends this analysis to the coordinator nodes. To complete the network description, Section 6 is focused on the central node, where the data collected by the coordinator nodes are 
received and properly represented and processed. Section 7 shows the network test, performed to evaluate both the complete system operation and the battery lifetime prediction model. Finally, Section 8 draws the conclusions.

\section{State of the Art}

Wireless sensor networks (WSN) have become a key environmental technology, with applications in intelligent agriculture $[1,2]$, diffuse greenhouse gas emission detection, smart composting monitoring and control [3], prevention and early detection of forest fires, or protection of critical infrastructure [4], among others. Nevertheless, currently available commercial WSN generic platforms need considerable improvements to meet the stringent requirements desirable for real open nature deployments, mainly in terms of complexity, cost, lifetime, and maintenance. As a result, the development of custom nodes to replace commercial ones to enhance performance is gaining strength. In this attempt, most results remain at laboratory test level, being rather hazardous to find implementations validated in an actual deployment scenario. In fact, focusing on the implementation and test of complete full-custom WSN dedicated to monitoring environmental parameters, only a few solutions can be found in the open literature [5-9]. However, the use of sophisticated and powerconsuming sensor devices, communication technologies, and protocols results in cost per node, energy requirements, transmit data rate, and required infrastructure which render these solutions unsuitable for battery-operated nodes deployed in natural areas [5-7]. In [5], IP video cameras are used for smoke and fire detection, which require high computational power, increasing the cost of both software and hardware; in [6], a Digital Enhanced Cordless Telecommunications (DECT) subnet is connected to a Global System Mobile (GSM) coordinator which communicates with a PC control center; in [7] a combination is proposed between ZigBee, Wi-Fi, and satellite communications. Solutions in $[8,9]$ focus the power optimization on efficient data communications: in [8], a wireless sensor network for microenvironmental application achieving efficient node data transmission is presented, showing a web-based tool for monitoring the deployed network, controlling data traffic, data processing and presentation, and remote node control and monitoring; in [9], the authors propose a network with a duty cycle management able to address stability in network connections and coverage at the same time. By synchronizing disjoint sensor subsets, the system can detect events which could be omitted by classical communication architectures, achieving a tradeoff between power consumption and event detection. This is useful in applications where event lost are not allowed, as defense tasks.

In this scenario, with power, node size, and cost per node being the restrictive conditions, specific novel designs are desirable to come across all the main challenges encountered in environmental projects. Accordingly, a custom low-power low-cost reliable WSN platform has been developed by authors for use in remote areas, which can be easily adapted to other monitoring systems. Based on a first prototype [10, 11], a number of modifications have been included in order to reach an enhanced WSN implementation mainly concerning the following: (i) the reduction of power consumption at both software and hardware levels; (ii) communication developments (different communication protocols, such as GSM/GPRS and IEEE 802.15.4, can be used, adding more versatility to the proposal); and (iii) time synchronization stability (a real-time clock fed by a supercapacitor is added for data integrity purposes).

Making use of this WSN architecture, this paper is focused on finding a reliable estimation of the lifetime; this is an essential information before deployment, especially in systems targeting remote environmental monitoring, which are designed for long-term unattended operation. Despite its importance, this key metric is usually parameterized through predictions that often use high level descriptions and/or ideal or basic battery models, simplistic assumptions which overestimate lifetime. Recent works progressively include more complete analysis but still exhibit limitations since they are mainly based on emulation methods, usually developed for particular platforms, which ultimately fail to consider real effects. The simulator mTOSSIM [12] evaluates lifetime in WSN but is restricted to platforms embedding the TinyOS operative system; power consumption is estimated at microcontroller instruction level, varying different network parameters (duty cycle, transmission frequency, and idle or sleep transceiver modes) and using battery models based on technical specifications. Similarly [13] is an emulatedbased method using MSPSim, which incorporates battery models with nonideal effects and a low-level description of the node hardware. In [14], authors consider the average current consumption over the different operation processes (transmission, reception, and idle or sleep states) and the corresponding consumption of the different platform elements in these states, but the battery model does not consider the influence of self-discharge, temperature, and recovery effects over the battery capacity. In addition, sensors are not included and the communication is only made between an end device and the coordinator, which are not real operation conditions. Following an experimental approach, in [15] authors use LR06 batteries of different brands and capacity in order to evaluate their influence on a node lifetime; however, the study is based on a node in listen state, considering neither transmission nor sensor measurement processes and thus not describing a real network behavior.

From the above discussion, it is clear that an accurate lifespan prediction model must consider the energy consumption of the sensor nodes under their different operation modes, in correlation with the real battery characteristics and the voltage regulation system, that is, including all hardware and software constraints. To do so, we consider that an experimental methodology is the best suitable choice. This is the goal of this paper: to attain a complete and practical lifetime model, based on the remaining energy battery level as standardized metric through an experimental methodology: using real nodes under real operating conditions, from the measured power consumption profile and the measured battery discharge pattern, the battery discharge rate is inferred. Preliminary results were introduced in [16], for a LiPo battery. This work deepens and completes the study, considering two 


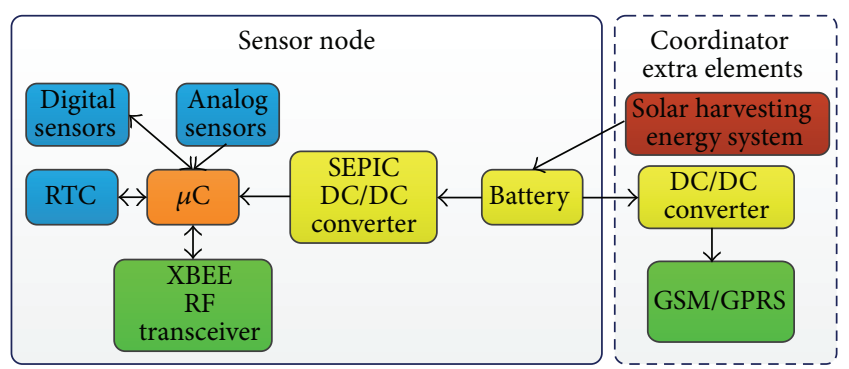

FIGURE 1: Sensor node block diagram (RTC: real-time clock; DC: Direct Current; RF: Radio Frequency; GSM: Global System for Mobile; GPRS: General Packet Radio Service).

different battery technologies, and validates results through a real outdoor deployment.

\section{WSN Description}

3.1. Sensor Node Architecture. The implemented basic node consists of the following core elements (Figure 1):

(i) An 8-bit low-power CMOS ATmegal281 microcontroller from Atmel that manages and synchronizes the node operation has been used.

(ii) An XBeePro transceiver operating in the $2.4 \mathrm{GHz}$ Industrial-Scientific-Medical (ISM) free RF band [17] has been included. By properly configuring the firmware parameters that control the transceiver operation, it is possible to drive all the network nodes to a low-power sleep state, thus providing a multihop energy-optimized communications protocol.

(iii) A real-time clock (RTC) model PCF2123 from NXP has been included. This component has been included to (i) enable arrangement of the collected data and (ii) detect errors in data transmission from the sequential information that it provides. This clock is programmed and activated before the network is deployed. It is firstly powered by a preloaded supercapacitor which stores the energy required for 5 days of RTC operation prior to its deployment, thus saving battery resources. In addition, powering the RTC by a supercapacitor allows replacement of node batteries without losing date information.

(iv) A power supply system is used consisting of a battery connected to a SEPIC DC-DC TPS61131 converter from Texas Instruments, which supplies the node components with the required energy and regulated voltage level $(3.3 \mathrm{~V})$. Also, a $100 \mathrm{mF}$ supercapacitor feeds the RTC for a further 7 days even when the node battery is fully discharged, thus avoiding the need to reprogram the clock.

(v) A $2.4 \mathrm{GHz} 7^{\prime \prime}$ and $5 \mathrm{dBi}$ dipole vertical polarization antenna has been used which allows a maximum outdoor range of about $1600 \mathrm{~m}$.

(vi) An IP65 standard protection box is used.
3.2. Sensor Types and Connectivity. To attain a versatile configuration, a custom low-voltage plug-and-play programmable electronic interface suitable for connecting both active and passive analog sensors has been developed. Programmability allows achieving an optimum reading performance for every connected sensor.

3.3. Network Communications. Depending on the WSN location, communication between the sensor nodes and the central node can be carried out in different ways: if the WSN is deployed in a region with mains infrastructure (such as indoor applications), nodes could be powered directly from the mains. In this case, energy-saving restrictions can be relaxed. However, in most cases sensor networks are not installed in regions with these infrastructures. The nodes therefore send the information to the coordinators using radiofrequency (RF) low-power communication protocols, most based on the IEEE 802.15.4 standard. Among these, some protocols [18] can drive the sensor nodes to a lowenergy mode but keep the router devices awake. These algorithms have several disadvantages: first, sensor distribution must be carefully selected to cover the transmission area of all the sensor nodes. Also, network scalability and selforganization are limited because of the need of performing the configuration of the nodes as end devices or routers before being installed. In addition, the operating life of the sensor network is clearly limited by the batteries of these router nodes.

These limitations are overcome by using the XBeePro transceiver including the DM-24 firmware, as will be shown next. Furthermore, this transceiver is fully pinout compatible with the WiFly $802.11 \mathrm{~b} / \mathrm{g}$ transceiver from Roving Networks [19] that allows the use of the same hardware node with high network versatility.

3.4. Power Management. The difficulty of battery replacement for sensors deployed in natural environments, joined to the unfavorable cost and size of most of battery recharge systems, makes lifespan a critical issue. Thus, in order to increase the network lifetime, the nodes use a Dynamic Power Management (DPM) technique. The microcontroller sets the digital components of the node (transceiver, smart sensors, and the microcontroller itself) into their corresponding lowpower modes when they are not active. Also, the polarization of analog components (like analog sensors, amplifiers, and filters) is controlled through an ADG701 digital switch from Analog Devices. Thus, when analog components are not in use, the microcontroller switches off the corresponding bias path. The node transceiver has five controllable power levels (Table 1) that allow optimizing the power consumption of the device. Finally, the IEEE 802.15.4 firmware programmed into the transceivers allows all the nodes to operate as Full Function Devices (FFD). In this way, the network router nodes are enabled to be in sleep mode without losing the network architecture, unlike the ZigBee specification [20, 21]. Note that a router which is permanently in the awake mode means a continuous current consumption of $67 \mathrm{~mA}$ (due mainly to the $\mathrm{RF}$ transceiver). So, if fed by a $2 \mathrm{Ah}$ battery like the one finally 
TABLE 1: XBeePro power levels.

\begin{tabular}{lc}
\hline Value of power level AT command & Power level $(\mathrm{dBm})$ \\
\hline 0 & 10 \\
1 & 12 \\
2 & 14 \\
3 & 16 \\
4 & 18 \\
\hline
\end{tabular}

used in this work, the operating life will be limited to only 30 hours.

Other power management widespread techniques are dynamic voltage scaling and dynamic frequency scaling. Dynamic voltage scaling (DVS) $[22,23]$ allows reducing the power consumption in low activity node modes. However, its implementation requires the use of additional electronics to provide the suitable different voltage supply values for the node components, increasing the final cost. In our case, due to the narrow voltage operation range of the XBee transceivers, whose bias voltage is limited between $2.8 \mathrm{~V}$ and $3.4 \mathrm{~V}$, the nodes include a SEPIC DC-DC converter that holds the voltage level between these limits. Reducing the bias voltage below $2.8 \mathrm{~V}$ resets the transceiver, thereby hardly limiting the DVS efficiency. In addition, in applications with a low duty cycle such as environmental monitoring, the required linear voltage converter needed for this method increases the power consumption in the low-power operation mode, degrading the node lifetime. On the other hand, the use of dynamic frequency scaling (DFS) $[24,25]$ allows reducing power consumption by dropping the system frequency according to the node operation requirements, though this technique is only suitable for microcontrollers that include specific integrated components. In some $\mu \mathrm{C}$ without these specifications, such as the one used in this work, it is possible to implement DFS using an additional RC circuit oscillator, which generates an output frequency value controlled by an iterative algorithm, but at a high cost in computational time and energy which drastically reduces its performance, and thus has been disregarded.

3.5. Commercial WSN Platforms Comparison. This basic sensor node component has been designed for flexibility, allowing for easy configuration as a sensor, sensor-router, or coordinator node. Table 2 shows a comparison of the proposed node with similar commercial devices. Crossbow nodes present lower energy requirements, but with 5 to 10 times lower transmission range. Therefore, for applications where sensors may be located several hundreds of meters apart, the need would arise to deploy additional router nodes, thereby increasing the network cost and complicating maintenance. On the other hand, the Waspmote shows similar outdoor and indoor transmission ranges. However, improved energy management is achieved in the proposed nodes, resulting in better overall performance. In addition, the use of a SEPIC enables biasing the nodes with a wider input voltage range.

\section{Lifetime Model}

Depending on what is under consideration, several different battery modeling methods can be found in the literature [26, 27]: physical, abstract, empirical, and mixed models, based on a combination of the previous modeling techniques. Physical modeling is the most accurate method, but at the cost of a high complexity that makes its practical application difficult. Abstract modeling describes battery operation based on electronic models, and it becomes highly suitable when the full system is to be simulated as a circuit. Finally, empirical modeling provides the simplest models, based on a numerical approach to describe the battery behavior. In this work, an empirical battery model has been developed. Following a systematic methodology, the proposed modeling considers the effect of the battery voltage reduction during the discharge process in real operation and thereby permits simple but realistic lifetime estimation. Besides, as several characteristics differ between rechargeable and nonrechargeable batteries, lifetime analysis has been performed using both battery type examples: a rechargeable 3.7 V-2000 mAh Lithium Polymer (LiPo) battery and $2 \times 1.5 \mathrm{~V}$ alkaline LR06 batteries with $2200 \mathrm{mAh}$ of charge.

The energy consumption of the basic sensor node is first analyzed to obtain the behavior in the different states over a working cycle. To perform this analysis, the node was configured as follows: (i) both analogue and smart digital sensors were connected: ambient parameters within the housing box are measured using two low-cost resistive analog sensors, NTC for temperature and Sencera H25K5A for relative humidity, while external parameters were measured using two digital smart sensors, Sensirion SHT11 to measure the relative humidity and Intersema MSB5540B for barometric pressure and temperature; (ii) a power transmission level of $18 \mathrm{dBm}$ (worst case) is selected; and (iii) the work cycle is set to a measuring time of $1.746 \mathrm{~s}$ every $900 \mathrm{~s}$ ( $15 \mathrm{~min}$ ), that is, a duty cycle of $0.18 \%$, a suitable choice for our target application. With this node configuration, sampling the power every $2 \mathrm{~ms}$ using a 2602A System SourceMeter (SMU) from Keithley Instruments connected to a PC through a USB-GPIB adapter, the power consumption profile of the full node presents three different levels: the first one $\left(\left(P_{1}\right), 898 \mathrm{~s}\right)$ corresponds to the system in sleep mode; the second one $\left(\left(P_{2}\right), 448 \mathrm{~ms}\right)$ corresponds to the parameters' measurement time, with microcontroller, transceiver, and sensors switched on; lastly, in the third one $\left(\left(P_{3}\right), 1.316 \mathrm{~s}\right)$, both sensors and microcontroller are switched off, whereas the transceiver remains on to complete the data transmission. In measurement (2) and transmission (3) stages, the power consumption is constant $\left(P_{2}=220 \mathrm{~mW}\right.$ and $P_{3}=235 \mathrm{~mW}$, resp.), whereas, in sleep mode (1), the current remains almost constant over all the battery operation range, with an average value below $i_{1}=12 \mu \mathrm{A}$.

Next, the battery behavior is characterized. The two selected alternatives, a rechargeable $3.7 \mathrm{~V}, 2000 \mathrm{mAh} \mathrm{LiPo}$ and a $2 \times 1.5 \mathrm{~V}$ LR06 $2200 \mathrm{mAh}$ alkaline battery, were 
TABLE 2: Sensor nodes comparison.

\begin{tabular}{|c|c|c|c|c|c|}
\hline CPU parameters & IRIS Crossbow & Micaz Crossbow & TelosB Crossbow & Waspmote Libelium & $\begin{array}{l}\text { Proposed node } \\
\text { (this work) }\end{array}$ \\
\hline \multicolumn{6}{|l|}{ Processor performance } \\
\hline Microcontroller & ATMega1281 & ATMega128L & MSP430 & ATMega1281 & ATMegal281 \\
\hline Number of bits & 8 bits & 8 bits & 16 bits & 8 bits & 8 bits \\
\hline Frequency & N/A & N/A & N/A & $8 \mathrm{MHz}$ & $4 \mathrm{MHz}$ \\
\hline Program flash memory & $128 \mathrm{kB}$ & $128 \mathrm{kB}$ & $48 \mathrm{kB}$ & $128 \mathrm{kB}$ & $128 \mathrm{kB}$ \\
\hline SRAM & $8 \mathrm{kB}$ & $4 \mathrm{kB}$ & $10 \mathrm{kB}$ & $8 \mathrm{kB}$ & $8 \mathrm{kB}$ \\
\hline EEPROM & $4 \mathrm{kB}$ & $4 \mathrm{kB}$ & $16 \mathrm{kB}$ & $4 \mathrm{kB}$ & $4 \mathrm{kB}$ \\
\hline Communications interfaces & UART, I2C, SPI & UART, I2C, SPI & UART, I2C, SPI, USB & UART, I2C, USB & UART, I2C, SPI \\
\hline Analog to digital converter & 10-bit ADC & 10-bit ADC & 12-bit ADC & 10-bit ADC & $\begin{array}{c}\text { 10-bit ADC } \\
\text { VFC }\end{array}$ \\
\hline Digital to analog converter & NO & $\mathrm{NO}$ & 12-bit DAC & NO & NO \\
\hline Current active mode & $8 \mathrm{~mA}$ & $8 \mathrm{~mA}$ & $1.8 \mathrm{~mA}$ & $9 \mathrm{~mA}$ & $2.4 \mathrm{~mA}^{*}$ \\
\hline Current sleep mode & $8 \mu \mathrm{A}$ & $<15 \mu \mathrm{A}$ & $5.1 \mu \mathrm{A}$ & $62 \mu \mathrm{A}$ & $<12 \mu \mathrm{A}^{*}$ \\
\hline \multicolumn{6}{|l|}{ RF transceiver } \\
\hline Frequency band & ISM $2.4 \mathrm{GHz}$ & ISM $2.4 \mathrm{GHz}$ & ISM $2.4 \mathrm{GHz}$ & ISM $2.4 \mathrm{GHz}$ & ISM $2.4 \mathrm{GHz}$ \\
\hline Outdoor range & $>300 \mathrm{~m}$ & $75 \mathrm{~m}$ to $100 \mathrm{~m}$ & $75 \mathrm{~m}$ to $100 \mathrm{~m}$ & $750 \mathrm{~m}$ to $1500 \mathrm{~m}$ & $750 \mathrm{~m}$ to $1500 \mathrm{~m}$ \\
\hline Indoor range & $>50 \mathrm{~m}$ & $20 \mathrm{~m}$ to $30 \mathrm{~m}$ & $20 \mathrm{~m}$ to $30 \mathrm{~m}$ & $60 \mathrm{~m}$ to $90 \mathrm{~m}$ & $60 \mathrm{~m}$ to $90 \mathrm{~m}$ \\
\hline Sensitivity & $-101 \mathrm{dBm}$ & $-94 \mathrm{dBm}$ & $-94 \mathrm{dBm}$ & $-100 \mathrm{dBm}$ & $-100 \mathrm{dBm}$ \\
\hline Max. Tx Power & $3 \mathrm{dBm}$ & $0 \mathrm{dBm}$ & $0 \mathrm{dBm}$ & $18 \mathrm{dBm}$ & $18 \mathrm{dBm}$ \\
\hline \multicolumn{6}{|l|}{ Current draw } \\
\hline Receive mode & $16 \mathrm{~mA}$ & $19.7 \mathrm{~mA}$ & $23 \mathrm{~mA}$ & $57.08 \mathrm{~mA}$ & $63.6 \mathrm{~mA}^{*}$ \\
\hline Maximum transmission current & $17 \mathrm{~mA}$ & $17.4 \mathrm{~mA}$ & N/A & $187.58 \mathrm{~mA}$ & $154 \mathrm{~mA}^{*}$ \\
\hline Sleep mode & NA & $1 \mu \mathrm{A}$ & $1 \mu \mathrm{A}$ & $120 \mu \mathrm{A}$ & $<12 \mu \mathrm{A}^{*}$ \\
\hline \multicolumn{6}{|l|}{ Power supply } \\
\hline Battery & $2 \times$ AA batteries & $2 \times$ AA batteries & $2 \times$ AA batteries & $\mathrm{N} / \mathrm{A}$ & LiPo battery \\
\hline External power & $2.7 \mathrm{~V}$ to $3.3 \mathrm{~V}$ & $2.7 \mathrm{~V}$ to $3.3 \mathrm{~V}$ & $\mathrm{~N} / \mathrm{A}$ & $3.3 \mathrm{~V}$ to $4.2 \mathrm{~V}$ & $1.8 \mathrm{~V}$ to $5 \mathrm{~V}$ \\
\hline
\end{tabular}

${ }^{*}$ Measures include sensors and RTC.

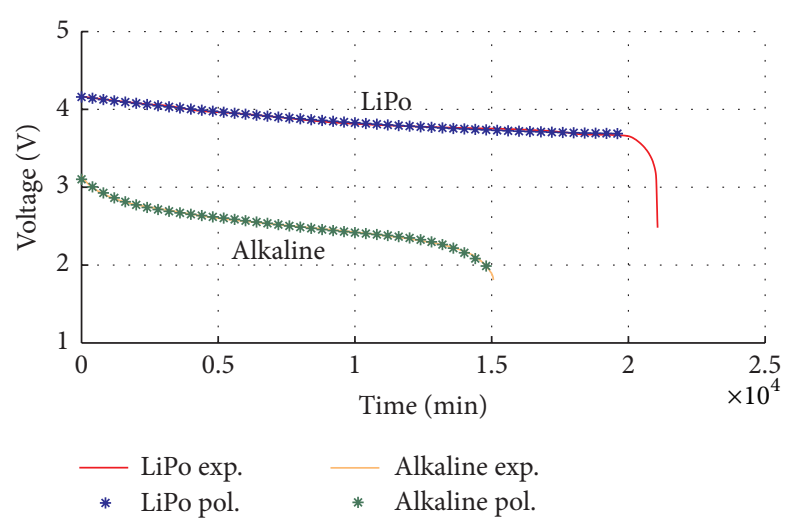

Figure 2: Experimental battery voltage (V) versus time (min) for a constant $220 \mathrm{~mW}$ discharge process.

discharged through the SEPIC converter at a constant power of $225 \mathrm{~mW}$ to match our working conditions. Figure 2 shows the resulting battery voltage drop versus time measured using an Agilent 34410A Multimeter connected to a PC through a USB-GPIB interface, sampling the battery voltage every
$60 \mathrm{~s}$. In the case of LiPo, for battery voltage ranges from 4.2 to $3.6 \mathrm{~V}$, the behavior can be modeled according to the approximated expression in (1), and once the battery voltage falls below $3.6 \mathrm{~V}$, its value quickly drops to $2.6 \mathrm{~V}$. For the alkaline batteries, operation fails when the voltage across both batteries drops to $1.8 \mathrm{~V}$, that is, the minimum operation voltage of the SEPIC converter. In this case, the related behavior model is given by (2):

$$
\begin{aligned}
& v(t)= 9.559 t^{2} \times 10^{-10}-4.275 t \times 10^{-5}+4.159 \\
& {[\mathrm{~V}, t \text { in minutes }], } \\
& v(t)=-2 t^{5} \times 10^{-15}+7.635 t^{4} \times 10^{-12}-1.066 t^{3} \\
& \times 10^{-8}+7.13 t^{2} \times 10^{-6}-2.71 t \times 10^{-3}+3.1
\end{aligned}
$$

$[\mathrm{V}, t$ in minutes].

Based on the results on Figure 2, Figure 3 shows the measured and modeled remaining energy versus battery voltage $v(t)$ for a maximum initial battery charge of $2 \mathrm{Ah}(120,000 \mathrm{mAmin})$ for the LiPo battery and $2.2 \mathrm{Ah}$ $(132,000 \mathrm{mAmin})$ for the alkaline one. In this paper, capacity 


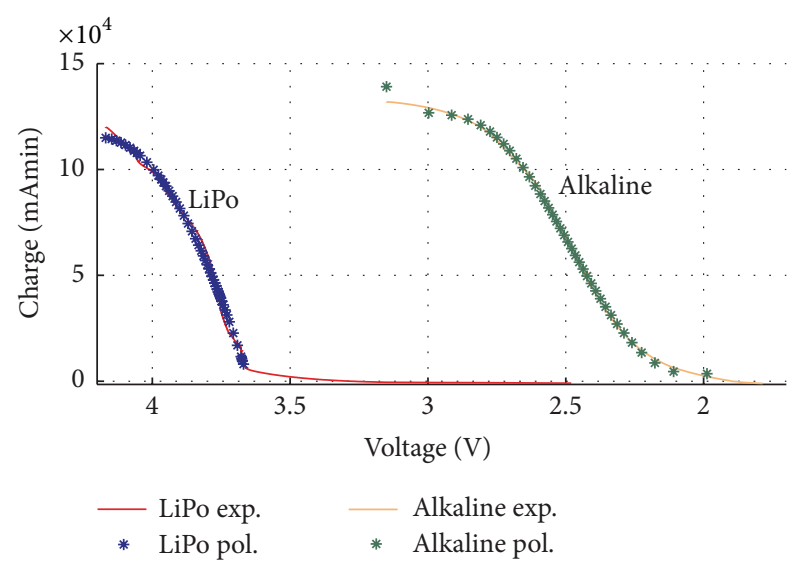

FIGURE 3: Experimental and polynomial fit for battery discharge (mAmin) versus battery voltage $(\mathrm{V})$. Initial battery energy: $2 \mathrm{Ah}$ (LiPo); $2.2 \mathrm{Ah}$ (alkaline).

(usually expressed in mAh) and energy (expressed in Joules) are used interchangeably.

Then, it is possible to determine the battery lifetime by evaluating the remaining charge as a function of the energy consumed by the full-node electronics. By assuming a 15minute work cycle and taking into account the three different power consumption profiles present over cycle $\left(P_{1}, P_{2}\right.$, and $\left.P_{3}\right)$, the battery discharge for a work cycle can be estimated according to

$$
C=i_{1} \int_{t_{0}}^{t_{1}} d t+\int_{t_{1}}^{t_{2}} i_{2}(t) d t+\int_{t_{2}}^{t_{3}} i_{3}(t) d t
$$

where $t_{0}-t_{1}$ are the limits of the sleep mode timespan, where $i_{1}$ is set to $15 \mu \mathrm{A}$ to ensure a conservative estimation of the lifetime and partially compensate possible effects not considered in the model that could worsen the battery operation, $t_{1}$ and $t_{2}$ define the limits of the measurement timespan, where $i_{2}=P_{2} / v(t)$, and $t_{2}$ and $t_{3}$ define the transmission timespan, where $i_{3}=P_{3} / v(t)$. Using this onecycle model, the evolution in the battery charge is iteratively calculated over time. Figure 4 shows the discharge process for the two considered battery types connected to a wireless node in full operation under our work conditions in view of different effects: ideal discharge (from (3)), considering maximum power transmission spikes and considering selfdischarge effects. In both cases, the discharge curve is highly linear. We next analyze power transmission spikes and selfdischarge dependences.

Discharge spikes have been measured using an Agilent Mixed Signal Oscilloscope 9409A connected to an Agilent N2783A current probe. Despite these discharge spikesdue to transmission operations-the operation lifetime is not significantly affected. For this reason, the blue and red lines are overlapped in both graphics in Figure 4. In fact, battery lifetime can be just slightly increased by selecting the suitable power transmission level (Table 1): in the maximum transmission level \#4 the power transmission spikes are $1 \mathrm{~W}$ high. This value is reduced by $165 \mathrm{~mW}$ per transmission spike for each power level reduction. Thus, assuming an ideal data

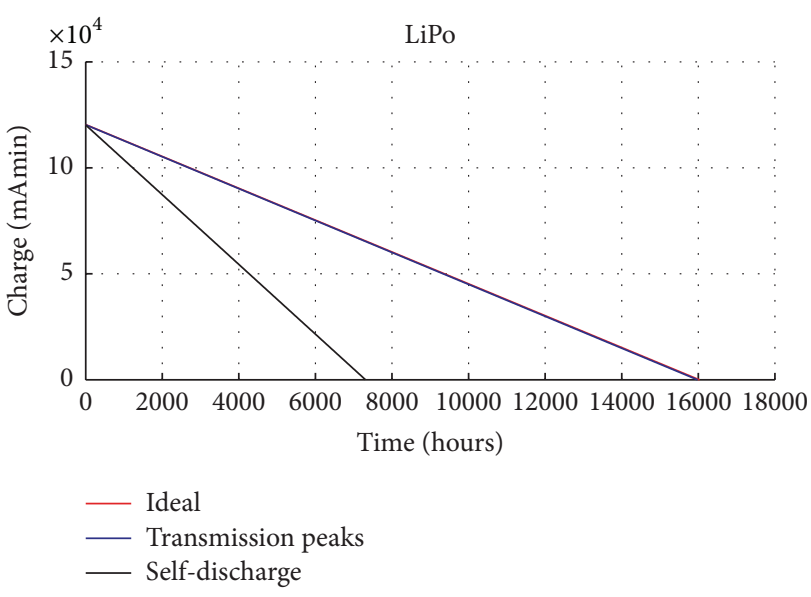

(a)

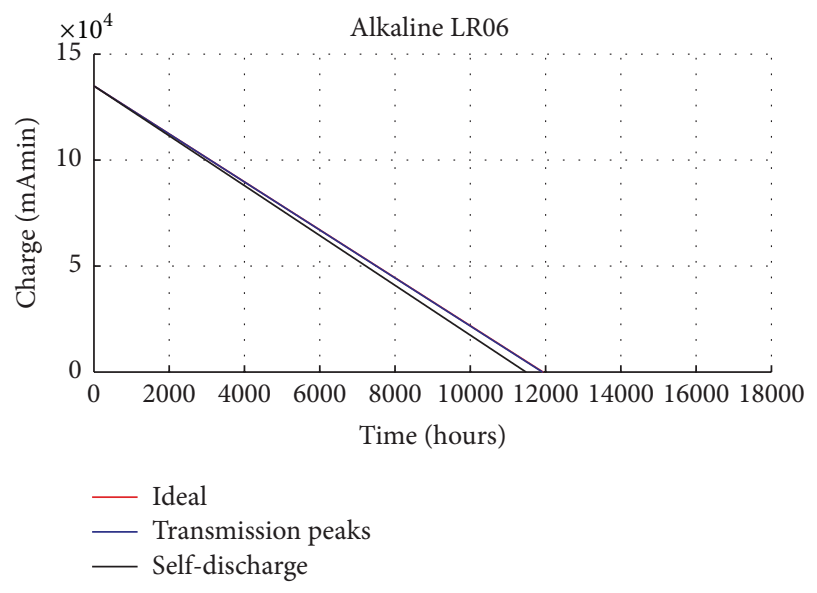

(b)

FIGURE 4: (a) LiPo charge evolution (mAmin) versus time (hours). (b) Alkaline battery charge evolution (mAmin) versus time (hours). In both cases ideal (red) and transmission (blue) graphs are overlapped.

transmission process for all data frames (without retries), the lifetime difference between the highest (4) and lowest (0) power level modes is less than 30 hours using the proposed duty cycle.

However, the repercussion of the self-discharge effect significantly influences lifetime, especially with rechargeable batteries. In the case of LiPo used in this work, it is 20\% [27]. Thus, depending on the application requirements it is very important to make a careful choice of battery. Considering all the main effects for LiPo and the alkaline batteries, the equations that represent lifetime are given by

$$
\begin{aligned}
& C(t)=-16.447 t+120210[\mathrm{mAmin}], \\
& C(t)=-11.7585 t+134970 \text { [mAmin], }
\end{aligned}
$$

respectively. The results obtained using (3) render a lifetime of around 670 days for a LiPo battery in ideal conditions. However, by considering the self-discharge effect this value, according to (4), falls to 300 days. Therefore, the use of this battery type requires a recharge system if longer operation 
time is expected. The same estimation is made with the alkaline batteries. In this case, the ideal lifetime is 500 days, less than the LiPo battery. In contrast, the lifetime, adding the effect of self-discharge, is reduced by only 20 days, giving an operating time of 480 days.

\section{Coordinator Node Architecture}

5.1. Preliminary Considerations. When using a GSM/GPRS coordinator, there are several options for transmitting data from a WSN to a central node. First, communication technology must be selected (the Internet mobile, phone call, short message service, etc.). Some authors propose the use of Internet mobile technology $[28,29]$. However, this choice requires high infrastructure and communication bandwidth, which may be impractical in many cases. Alternatively, the short message service (SMS) requires much less infrastructure and bandwidth, making it suitable for such applications. In addition, all providers of mobile phone services provide SMS as a basic service without special subscription. Furthermore, this solution can be easily adopted using a portable satellite phone service and can therefore be used worldwide.

For an efficient network sensor management, the optimum maximum network size must be estimated. Although the number of motes connected to a single coordinator node in a WSN is theoretically unlimited, energy constraints and cost make it unfeasible. For most applications, networks consisting of 100-200 nodes connected to a single coordinator are adequate for monitoring large areas. If more nodes are to be connected, further coordinator nodes can be added to form subnetworks to send the information from the new items to the central node.

The data transmission protocol to the central node poses an important restriction: hardware limitations in GSM modules will limit the data size to be sent through an SMS. The module used in this work is a GM862 from Telit $[30,31]$, whose bias voltage ranges from 3.4 to $4.2 \mathrm{~V}$ with maximum current peaks of $2 \mathrm{~A}$. Assuming that each sensor node collects the information from 16 sensors and a sensor measurement is represented by 2 bytes, the sensor data transmitted by a node to its coordinator are stored in 32 bytes. Also, a sensor node sends 7 bytes corresponding to the time and date information provided by the RTC module. Therefore, including other additional parameters such as node address, the total information forwarded from a sensor node to its coordinator is sent in 50 bytes. In addition, the GSM module only transmits printable characters; thus, the data bytes received, represented as hexadecimal values, are converted to ASCII characters. Then, the coordinator node concatenates the information before the transmission through the GSM. According to the data size, information is split into the required number of SMS frames and sent consecutively. Figure 5 shows an SMS complete data frame. Because the data received by the network coordinator could require several concatenated SMSs, the first bytes of each SMS identify the position of the SMS in the full SMSs thread. The next bytes indicate the length of the total information sent by the network coordinator, while the following information corresponds to the length of the information sent by one

\begin{tabular}{|c|c|c|c|c|c|c|}
\hline $\begin{array}{c}\text { Position } \\
\text { SMS }\end{array}$ & $\begin{array}{c}\text { Total } \\
\text { length }\end{array}$ & $\begin{array}{c}\text { Partial } \\
\text { length }\end{array}$ & $\begin{array}{c}\text { Frame } \\
\text { data 1 }\end{array}$ & $\begin{array}{c}\text { Partial } \\
\text { length }\end{array}$ & $\begin{array}{c}\text { Frame } \\
\text { data 2 }\end{array}$ & \\
EOT \\
\hline
\end{tabular}

FIGURE 5: Data collection format sent by the GSM coordinator through Telit GM862 module.

node. The rest of the SMS frame contains the information collected by the corresponding sensor nodes, including its identification and the sensor readings. The last byte is an end of transmission (EOT) code.

Two different ways exist to store the data received from the sensor nodes in the coordinator before forwarding it to the central node: by using either the memory of the node microcontroller or that of the GSM module. Although the data size is similar in both cases, the program required to send data via SMS from the microcontroller memory is simpler. Therefore, to simplify the coordinator setup and ensure correct operation of the system, the data are stored directly in the microcontroller memory, thereby limiting the number of sensor nodes to 100 ( $<90$ SMS per data transfer).

5.2. Hardware Design. The core architecture of a coordinator node is the same as that of a sensor node (see Figure 1). However, the connection of the sensor device to a GSM module requires certain changes in the power management system and the software programmed into the microcontroller. As the value of the GSM bias voltage is higher than that of the rest of the node components, the $3.7 \mathrm{~V}$ Lithium Polymer battery is selected as the energy source. It feeds the GSM module directly, whereas the rest of the electronics are powered through the SEPIC DC-DC converter that regulates the voltage to the suitable $3.3 \mathrm{~V}$.

The node microcontroller is responsible for arranging the data provided by the nodes in the network to be sent in the SMSs, starting the GSM module, and sending the messages. Communication between the node microcontroller and the GSM module is performed through a serial protocol, using a Universal Asynchronous Receiver-Transmitter (UART) port. Because of the difference in the bias voltage of the node microcontroller $(3.3 \mathrm{~V})$ and the GSM module (from 3.4 to $4.2 \mathrm{~V}$ ), signal accommodation is required: the microcontroller receiving port is able to accurately read the data sent by the communications module; however, the receiving port of the GSM module needs voltage attenuation in the logic signals for a suitable interface.

5.3. Microcontroller Software. Control of the GSM module operation is performed by the coordinator node microcontroller by means of a Finite State Machine (FSM). This FSM controls the system evolution through the different defined states, from the start pulse of $2 \mathrm{~s}$, which turns on the device, until the same pulse is sent for shutdown. The FSM also allows monitoring the correct transmission of the GSM AT commands, thus avoiding undesired states of the system that could drive the communications module to a malfunction. Accordingly, the FSM evolves to the next state only when the GSM module confirms that the command has been successfully processed. The FSM flowchart is shown 


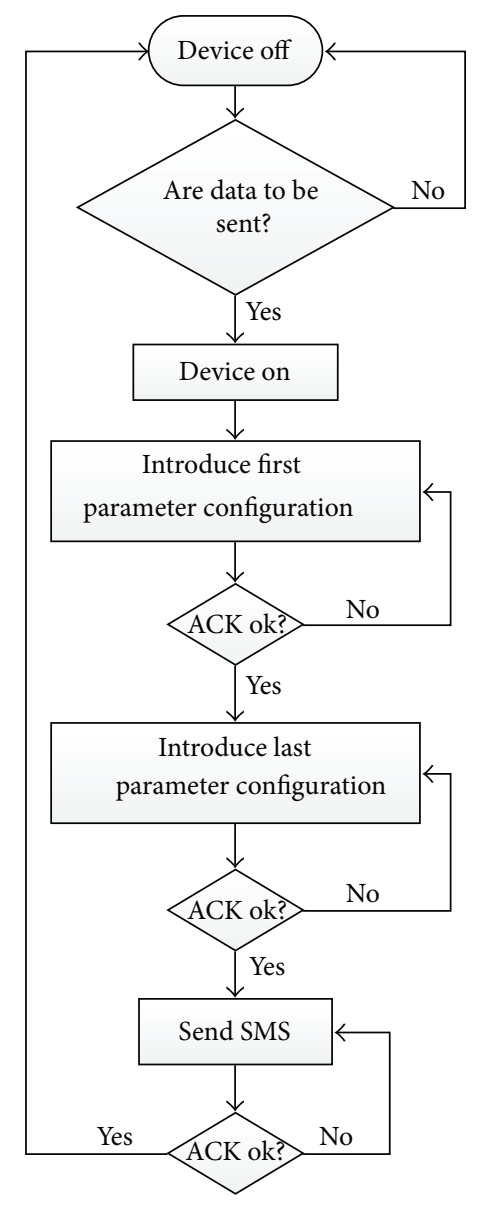

FIGURE 6: Coordinator node $\mu$ C FSM flowchart.

in Figure 6. In a measurement operation, once the wake-up signal is given by the transceiver to the node components, the node microcontroller first checks if there are data to be sent to the network controller via SMS. In this case, the $\mu \mathrm{C}$ sends a wake-up pulse to the GSM device. Data transmission from the $\mu \mathrm{C}$ to the GSM module starts when the measurements of the corresponding node sensors are fully collected by the node coordinator. Parameters of the GSM connection to the service provider (e.g., pin number) are sequentially sent to the module from the node microcontroller, verifying the proper reception by the corresponding acknowledgment (ACK) signals. When the module configuration is completed, the microcontroller sends the data to be transmitted to the GSM, which then starts the corresponding transmission. If the process is successfully executed, the GSM module is switched off until a new data transmission is to be started.

Parallel to this supervision method a timer controls the confirmation timeout of the communications module. If this time is exceeded, the system restarts the FSM state and repeats the process of sending the command. This time must be long enough to avoid the loss of the acknowledgement responses sent by the GSM module when a command is properly received and processed. We have found that a timeout between 1 and $2 \mathrm{~s}$ is adequate. A module reset has also been implemented, which the microcontroller activates when the module stops answering; however, this GSM reset must be avoided whenever possible as it does not send a disconnection notice to the GSM network.

5.4. Power Management. The GSM module power consumption constitutes the main reduction factor on the coordinator node battery. It includes a standby mode in which it is possible to receive SMS. However, if the time between two consecutive data transmissions is set over the minute, the power consumption in this standby mode will be higher than the consumption for the process of switching on the module, connecting to the GSM network, transmitting the data, and switching off. Typically, in environmental applications, the time communication between two consecutive data transmissions is usually of several minutes, and thus we chose this second option of operation for the GSM module. Estimating the consumption in an SMS transmission cycle by averaging the measured consumptions over the different states from the module startup to switch off, with the same sensors as the previously reported sensor node, the coordinator node reaches $3.11 \mathrm{~mA}$ consumption per cycle due to the presence of the GSM module. If the node is powered our LiPo selected battery (2000 mAh) lifetime is below one month; for a $6000 \mathrm{mAh}$ battery, similar in size to those in the sensor nodes, the lifetime will be below 3 months. Therefore an additional energy harvesting system (Figure 1) is required to extend it.

\section{Central Node Architecture}

6.1. GSM Data Receiver Module. For the central node, where data are received, consumption is not a main issue. In this case, the module is connected to a PC, which subsequently processes the information. Therefore, an energy source must be available for the PC and, hence, for the GSM module, that can be switched on permanently.

The GSM receiver hardware has been implemented following the manufacturer's specifications and using only the relevant features for this application, thus minimizing the design requirements. The power supply complies with the requirements of the module: an output voltage between 3.4 and $4.2 \mathrm{~V}$, providing a maximum peak current of 2 A.

6.2. PC Control. A software application has been specifically developed for SMS reception, data unthreading, and information processing and display. This software has been implemented using the Graphical User Interface Development Environment (GUIDE) from MATLAB. The application can interpret the information received by the coordinator node independently of the communication technology; namely, it can process the data irrespective of whether it is received by IEEE 802.15.4, SMS, or WLAN. It allows selecting the type of transmission protocol, making it possible to both receive or send data and change the network settings. Moreover, the application can arrange the data collected by various sensor types and locations (nodes) using the accurate time stamp provided by the RTC chip found in the hardware of the sensor nodes. 


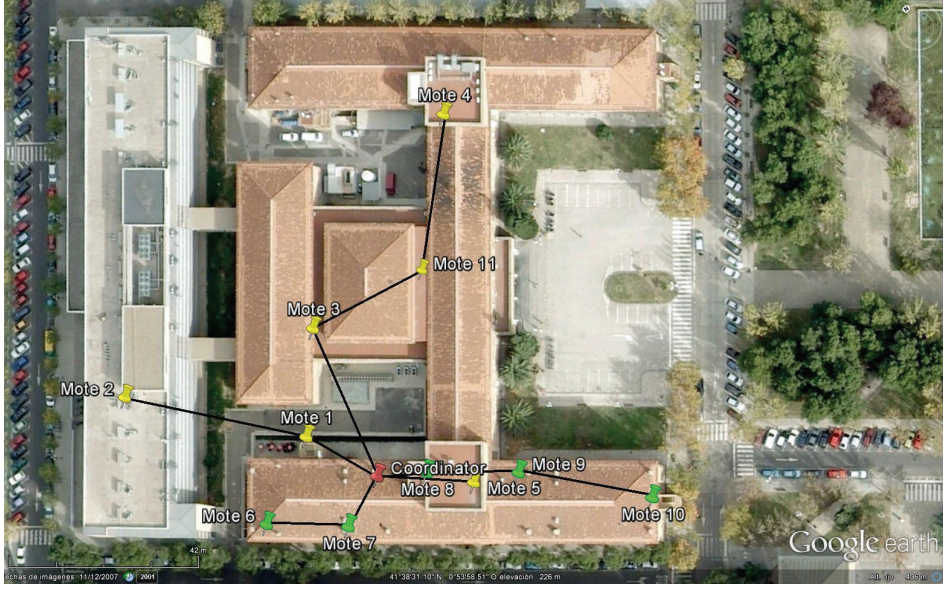

(a)

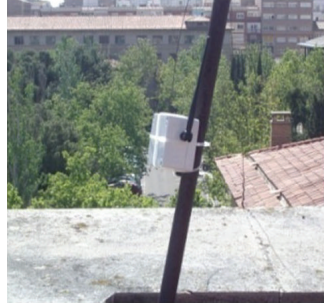

(b)

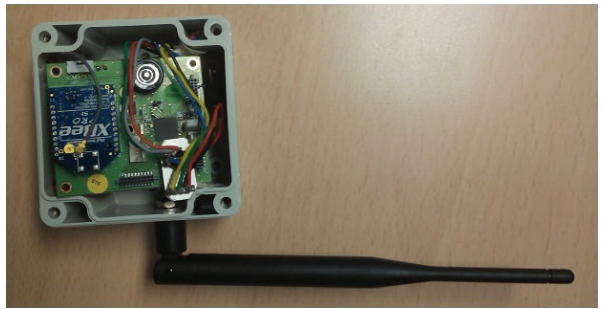

(c)

FIGURE 7: (a) Test sensor network deployment. Yellow thumbtacks correspond with outdoor nodes and green thumbtacks with indoor nodes. The red thumbtack is the network coordinator (scale: 1:2500, (c) 2013 Google); (b) node deployment detail; and (c) sensor node architecture.

Making the desired selection, this interface allows data from different nodes of the sensor network to be shown. It is also possible to choose the parameters to be monitored (relative humidity inside or outside the node housing box, temperature, etc.). In addition, a collection of historic data from a specific node or a group of them can be displayed. Finally, the interface allows changing different network parameters (measurement frequency, maximum network hops, mesh retries, node reset, power level, node disconnection, etc.), thus adapting the network architecture to the evolution of the environmental conditions.

\section{Network Test}

To validate the WSN operation, a network prototype consisting of 11 sensor nodes was deployed for a 6-month period at the Faculty of Science at the University of Zaragoza. The node distribution is shown in Figure 7. Green thumbtacks represent nodes located inside the building, whereas yellow thumbtacks are nodes located on the roof. The network coordinator is represented by a red thumbtack. Table 3 shows the complete deployment network characteristics and information on the nodes' spatial distribution (location, height, line or non-line of sight, power transmission, and role). All match the previously performed analysis; that is, each sensor node includes an NTC thermistor and an analog Sencera H25K5A sensor for monitoring temperature and relative humidity in the sensor housing box; external parameters are monitored through a Sensirion SHT11 smart relative humidity and temperature sensor and an Intersema MS5540B smart pressure sensor. The duty cycle is of $900 \mathrm{~s}$ (15 min), with $1.746 \mathrm{~s}$ awake time (including measurement and transmission operations), and power supply is provided by a $3.7 \mathrm{~V} \mathrm{LiPo}$ battery through a SEPIC DC-DC TPS61131 with fixed $3.3 \mathrm{~V}$ output.

Before deploying the network, sensor nodes are configured to assure a fast network connection: maximum transmission power level $(18 \mathrm{dBm})$, sleep support mode (which allows setting all the nodes to sleep mode), and low sleep period. After deploying the nodes, the central node starts a searching process. Once a node is associated to the network, its parameters are set to their final values (sleep and wake periods, maximum network hops, etc.).

The tested network revealed that the success frame transmission ratio is highly dependent on the suitable configuration of the transceiver firmware. The inclusion in the transmitted data of the timing information provided by the RTC included in the node electronics enabled both time arrangement of the received frames and the determination of the missing messages. As the date of every node transmission is made known through its RTC, the actual date provided by the PC allows organizing the node transmissions in time. Also, as the central node knows the number of nodes in the network, it is possible to discover any unsuccessful transmissions by comparing the number of transmissions received from other nodes following the time corresponding 
TABle 3: Deployment characteristics.

\begin{tabular}{|c|c|c|c|c|c|}
\hline Sensor node & Location & High & Line of sight & Power level & Role \\
\hline Coordinator & Indoor & Second floor & - & $4(18 \mathrm{dBm})$ & Coordinator \\
\hline Mote 1 & Outdoor & Second floor & LOS $^{*}$ & $4(18 \mathrm{dBm})$ & Router \\
\hline Mote 2 & Indoor & Fifth floor & LOS & $4(18 \mathrm{dBm})$ & End device \\
\hline Mote 3 & Indoor & Third floor & LOS & $4(18 \mathrm{dBm})$ & Router \\
\hline Mote 4 & Indoor & Fourth floor & NLOS** & $4(18 \mathrm{dBm})$ & End device \\
\hline Mote 5 & Indoor & Fourth floor & NLOS & $4(18 \mathrm{dBm})$ & End device \\
\hline Mote 6 & Outdoor & Second floor & NLOS & $4(18 \mathrm{dBm})$ & End device \\
\hline Mote 7 & Outdoor & Second floor & NLOS & $4(18 \mathrm{dBm})$ & Router \\
\hline Mote 8 & Outdoor & Second floor & NLOS & $4(18 \mathrm{dBm})$ & Router \\
\hline Mote 9 & Outdoor & Second floor & NLOS & $4(18 \mathrm{dBm})$ & Router \\
\hline Mote 10 & Outdoor & Second floor & NLOS & $4(18 \mathrm{dBm})$ & End device \\
\hline Mote 11 & Indoor & Third floor & NLOS & $4(18 \mathrm{dBm})$ & Router \\
\hline
\end{tabular}

* LOS: line of sight; ${ }^{* *}$ NLOS: non-line of sight.

TABle 4: Package Error Ratio.

\begin{tabular}{lccc}
\hline Indoor nodes & Rate (\%) & Outdoor nodes & Rate (\%) \\
\hline LOS (end device) & 0.97 & LOS (end device) & 0.98 \\
LOS (router) & 1 & LOS (router) & 1 \\
NLOS (end device) & 2.36 & NLOS (end device) & 1.3 \\
NLOS (router) & 2.6 & NLOS (router) & 1.4 \\
\hline
\end{tabular}

to the maximum number of transmission retries per node (mesh retries). Table 4 shows the average Package Error Ratio (PER) for the presented configuration with mesh retries limited to three and the maximum network hops to six.

The environmental monitoring results obtained in node \#1 over two days are shown in Figure 8. Figure 8(a) corresponds to the temperature measured in the environment (blue) and inside the container (red). Figure 8 (b) is the relative humidity in the environment (blue) and inside the container. Finally, Figure 8(c) shows the barometric pressure. As shown in the figures, the inside relative humidity is almost constant. This indicates that the prototype is adequately isolated from the environment, which is the desired effect using an IP 65 box container. In the same way, the temperature evolution presents similar values inside and outside, the difference given by the slower inertia presented by the inbox sensor at temperature variations.

The suitability of the battery discharge model developed in this work was tested by monitoring the battery energy evolution in nodes \#1, \#6, \#7, \#8, and \#11 (Figure 9) for one month. Compared to measurements, the developed model (including transmission spikes and battery self-discharge) shows an average error of below 5\% for outdoor nodes and $10 \%$ for indoor nodes. Differences between data and discharge model are due to not only the model errors but also the data sending retries, not included in the model. In fact, note that indoor node batteries show less adequate model fitting. This difference is mainly due to the number of data frames lost. In this case, the node retries required to send lost frames which are set by the mesh retries transceiver parameter consume more battery energy, thereby reducing its operating life.

Different tests have been carried out to check the effects of inclusion of new nodes in the network already deployed, working and achieving successful results. The network reconfiguration due to the changes in the topology uses a work cycle; that is, during the first cycle after deployment of the new nodes, the system registers their inclusion; then data begins to be sent to the coordinator in the next cycle. Additionally, the effects on battery life for a node acting as a router for up to 10 sensor nodes have been analyzed. Using the previously specified network parameters, the measurementtransmission energy profile presented by a router is similar to that of a sensor node but presents additional power peaks whose influence on the average power consumption is below $1.5 \%$ per routed node. Therefore, more powerful batteries must be considered with mesh topologies where nodes close to the coordinator are acting as routers for several sensor devices.

\section{Conclusions}

This paper presents a general approach to accomplish a simple but reliable lifetime prediction of a battery-powered wireless sensor node. For this, an experimental based methodology including all hardware and software constraints has been developed, using a custom low-power low-cost reliable WSN platform. Thanks to it, prior to deployment in the field, it is possible to estimate the application's lifetime, while adopting strategies (adjustment of parameters such as power transmission level and duty cycle) to increase the network lifetime. The analysis of different kind of batteries allows comparing the best selection for each network deployment, depending on the application. Finally, through a real deployment, we have been able to test the complete system, validating the lifetime predicting model, while the WSN has proved to be robust for environmental monitoring. One shortcoming that has emerged is that, due to the high energy consumption of the GSM modules included in the coordinator units, their operating life is rather restricted. Thus, present studies are 


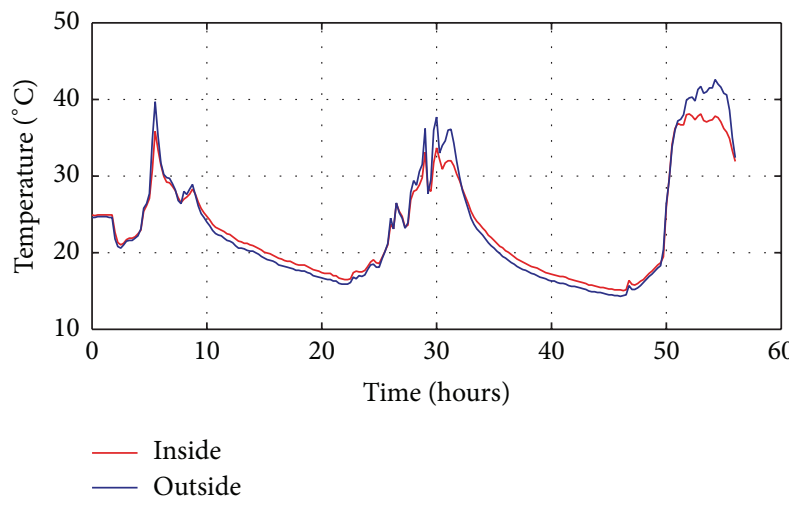

(a)

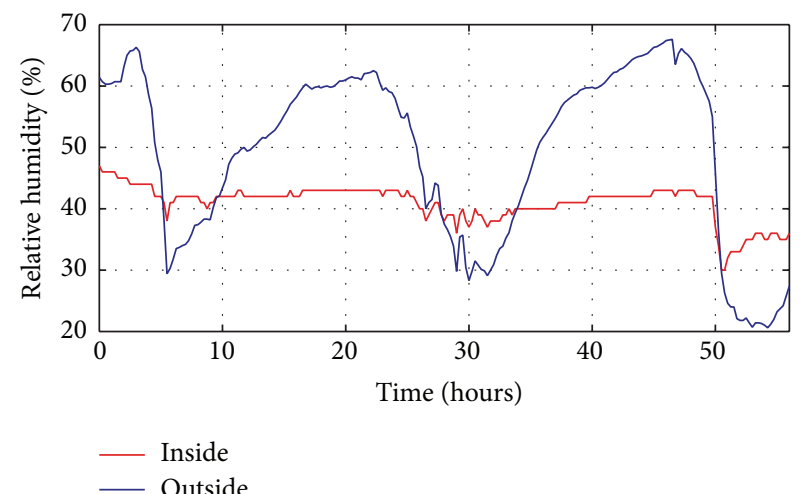

(b)

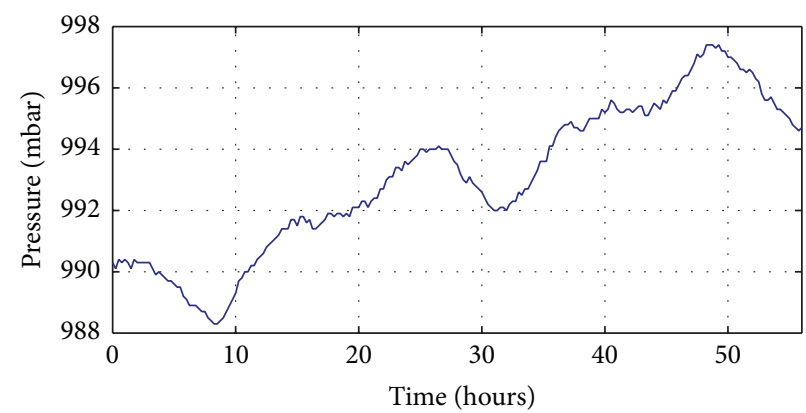

(c)

Figure 8: Data acquisition for node \#1 inside (red) and outside (blue) the box: (a) temperature, (b) relative humidity, and (c) barometric pressure.

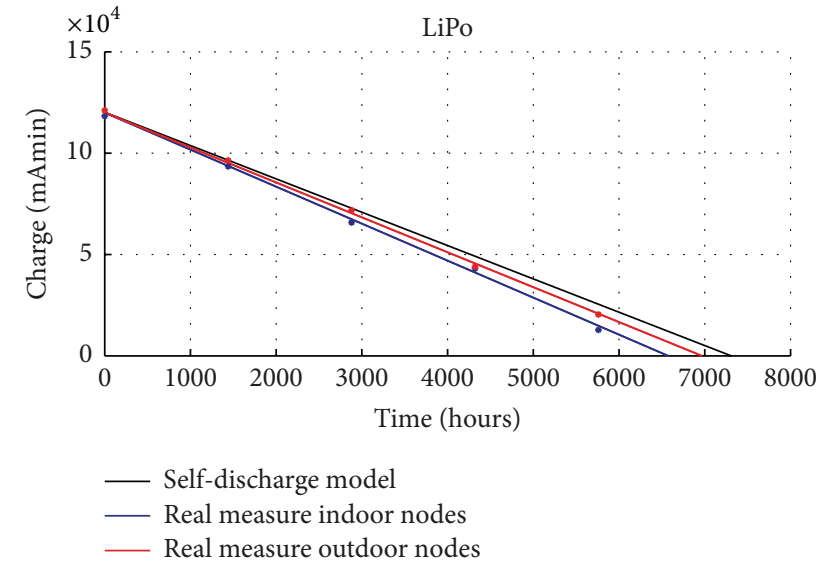

FIGURE 9: Lifetime with ideal self-discharge model, comparing with lifetime measures for indoor and outdoor nodes.

oriented towards the development of an efficient energy harvesting system for the coordinator node in order to increase network autonomy.

\section{Conflict of Interests}

The authors declare that the work presented in this paper has no conflict of interests.

\section{Acknowledgment}

This work was supported in part by MICINN-FEDER (TEC2012-30802, TEC2014-52840-R, and TEC2015-65750$\mathrm{R})$.

\section{References}

[1] P. Mariño, F. P. Fontán, M. Á. Domínguez, and S. Otero, "An experimental ad-hoc WSN for the instrumentation of biological models," IEEE Transactions on Instrumentation and Measurement, vol. 59, no. 11, pp. 2936-2948, 2010.

[2] J. Gutierrez, J. F. Villa-Medina, A. Nieto-Garibay, and M. A. Porta-Gandara, "Automated irrigation system using a wireless sensor network and GPRS module," IEEE Transactions on Instrumentation and Measurement, vol. 63, no. 1, pp. 166-176, 2014.

[3] O. Casas, M. López, M. Quílez et al., "Wireless sensor network for smart composting monitoring and control," Measurement, vol. 47, no. 1, pp. 483-495, 2014.

[4] L. Buttyán, D. Gessner, A. Hessler, and P. Langendoerfer, "Application of wireless sensor networks in critical infrastructure protection: challenges and design options," IEEE Wireless Communications, vol. 17, no. 5, pp. 44-49, 2010.

[5] J. Lloret, M. Garcia, D. Bri, and S. Sendra, "A wireless sensor network deployment for rural and forest fire detection and verification," Sensors, vol. 9, no. 11, pp. 8722-8747, 2009.

[6] A. Flammini, D. Marioli, E. Sisinni, and A. Taroni, "Environmental telemonitoring: a flexible GSM-DECT-based solution," 
IEEE Transactions on Instrumentation and Measurement, vol. 56, no. 5, pp. 1688-1693, 2007.

[7] K. Liolis, S. Pantazis, V. Gennatos, S. Costicoglou, and I. Andrikopoulos, "An automated fire detection and alerting application based on satellite and wireless communications," in Proceedings of the 5th Advanced Satellite Multimedia Systems Conference and the 11th Signal Processing for Space Communications Workshop (SPSC '10), pp. 270-277, IEEE, Cagliari, Italy, September 2010.

[8] X. Cao, J. Chen, Y. Zhang, and Y. Sun, "Development of an integrated wireless sensor network micro-environmental monitoring system," ISA Transactions, vol. 47, no. 3, pp. 247$255,2008$.

[9] S. He, J. Chen, and Y. Sun, "Coverage and connectivity in dutycycled wireless sensor networks for event monitoring," IEEE Transactions on Parallel and Distributed Systems, vol. 23, no. 3, pp. 475-482, 2012.

[10] D. Antolín, A. Bayo, N. Medrano, B. Calvo, and S. Celma, "Data management of wireless sensor network implemented in rural environments with SMS communication protocol," in Proceedings of the IEEE Sensors Conference, pp. 2002-2005, IEEE, Limerick, Ireland, October 2011.

[11] D. Antolín, A. Bayo, N. Medrano, B. Calvo, and S. Celma, "WubiNet: a flexible WSN for applications in environmental monitoring," in Proceedings of the IEEE International Instrumentation and Measurement Technology Conference (I2MTC '12), pp. 2608-2611, IEEE, Graz, Austria, May 2012.

[12] J. M. Mora-Merchan, D. F. Larios, J. Barbancho, F. J. Molina, J. L. Sevillano, and C. León, "mTOSSIM: a simulator that estimates battery lifetime in wireless sensor networks," Simulation Modelling Practice and Theory, vol. 31, pp. 39-51, 2013.

[13] W. Dron, S. Duquennoy, T. Voigt, K. Hachicha, and P. Garda, "An emulation-based method for lifetime estimation of wireless sensor networks," in Proceedings of the IEEE International Conference on Distributed Computing in Sensor Systems (DCOSS '14), pp. 241-248, IEEE, Marina Del Rey, Calif, USA, May 2014.

[14] F. Kerasiotis, A. Prayati, C. Antonopoulos, C. Koulamas, and G. Papadopoulos, "Battery lifetime prediction model for a WSN platform," in Proceedings of the 4th International Conference on Sensor Technologies and Applications (SENSORCOMM '10), pp. 525-530, IEEE, Venice, Italy, July 2010.

[15] H. A. Nguyen, A. Förster, D. Puccinelli, and S. Giordano, "Sensor node lifetime: an experimental study," in Proceedings of the 9th IEEE International Conference on Pervasive Computing and Communications Workshops (PERCOM Workshops '11), pp. 202-207, Seattle, Wash, USA, March 2011.

[16] D. Antolin, N. Medrano, and B. Calvo, "Analysis of the operating life for battery-operated wireless sensor nodes," in Proceedings of the 39th Annual Conference of the IEEE Industrial Electronics Society (IECON '13), pp. 3883-3886, IEEE, Vienna, Austria, November 2013.

[17] XBEE/XBEE-PRO DigiMesh 2.4 OEM RF Modules Datasheet, Digi International, 2008.

[18] S. Farahani, Zigbee Wireless Networks and Transceivers, Newnes, 2006.

[19] RN-XV Data Sheet, RN-XV-DS v0.3, 2011, http://www.rovingnetworks.com/.

[20] ZigBee Alliance, "ZigBee specification,” Document 053474r17, 2008.

[21] ZigBee Alliance, "ZigBee-2007 layer PICS and stack profiles ZigBee," Document 08006r03, 2008.
[22] R. Cheour, K. Lahmar, and M. Abid, "Evolution of wireless sensor networks and necessity of power management technique," in Proceedings of the Faible Tension Faible Consommation (FTFC '11), pp. 75-78, IEEE, Marrakech, Morocco, June 2011.

[23] U. Kulau, F. Büsching, and L. Wolf, "A node's life: increasing WSN lifetime by dynamic voltage scaling," in Proceedings of the 9th IEEE International Conference on Distributed Computing in Sensor Systems (DCoSS '13), pp. 241-248, IEEE, Cambridge, Mass, USA, May 2013.

[24] V.-T. Hoang, N. Julien, and P. Berruet, "Increasing the autonomy of wireless sensor node by effective use of both DPM and DVFS methods," in Proceedings of the IEEE Faible Tension Faible Consommation (FTFC '13), pp. 1-4, IEEE, Paris, France, June 2013.

[25] S. Park, A. Savvides, and M. B. Srivastava, "Battery capacity measurement and analysis using lithium coin cell battery," in Proceedings of the International Symposium on Low Electronics and Design (ISLPED '01), pp. 382-387, San Francisco, Calif, USA, August 2001.

[26] R. Rao, S. Vrudhula, and D. Rakhmatov, "Battery modeling for energy-aware system design," IEEE Computer, vol. 36, no. 12, pp. 77-87, 2003.

[27] Data Sheet, "Li-polymer battery packs," Specification Type $5854602000 \mathrm{mAh}, 2006$.

[28] A. R. Al-Ali, I. Zualkernan, and F. Aloul, "A mobile GPRSsensors array for air pollution monitoring," IEEE Sensors Journal, vol. 10, no. 10, pp. 1666-1671, 2010.

[29] C. K. Harnett, "Open wireless sensor network telemetry platform for mobile phones," IEEE Sensors Journal, vol. 10, no. 6, pp. 1083-1084, 2010.

[30] Telit, Telit Modules Software User Guide, 1vv0300784 Rev.3, Telit, Rome, Italy, 2010.

[31] GM862 Family Hardware User Guide, 2009, http://www.roundsolutions.com/techdocs/GM862/GM862-QUAD_PY_Hardware User_Guide_rl.pdf. 


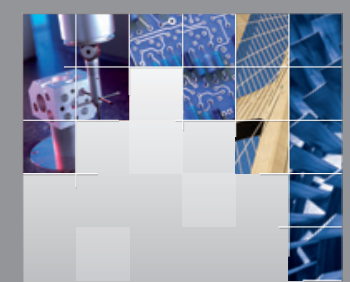

\section{Enfincering}
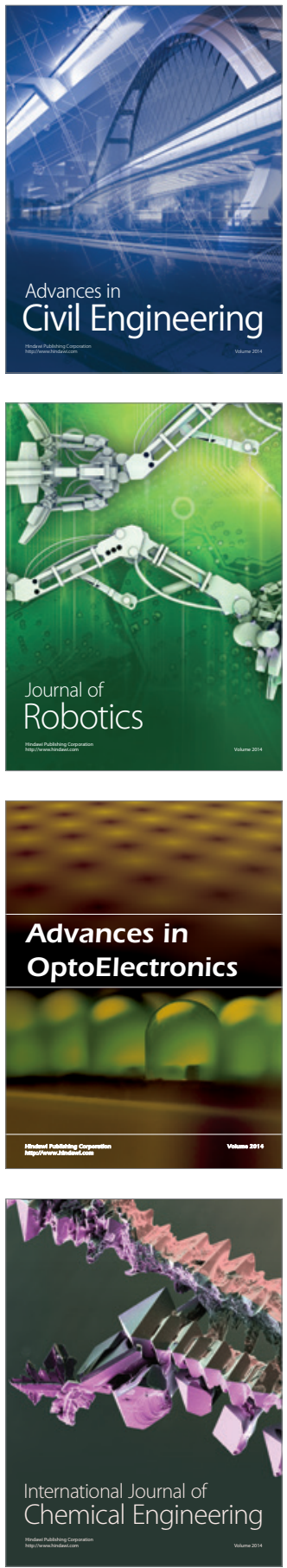

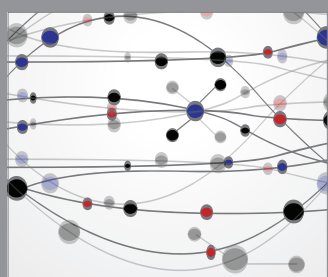

The Scientific World Journal

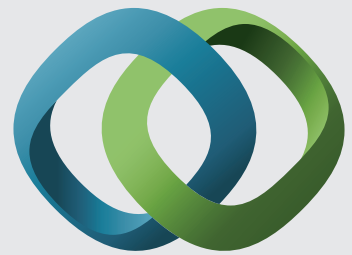

\section{Hindawi}

Submit your manuscripts at

http://www.hindawi.com
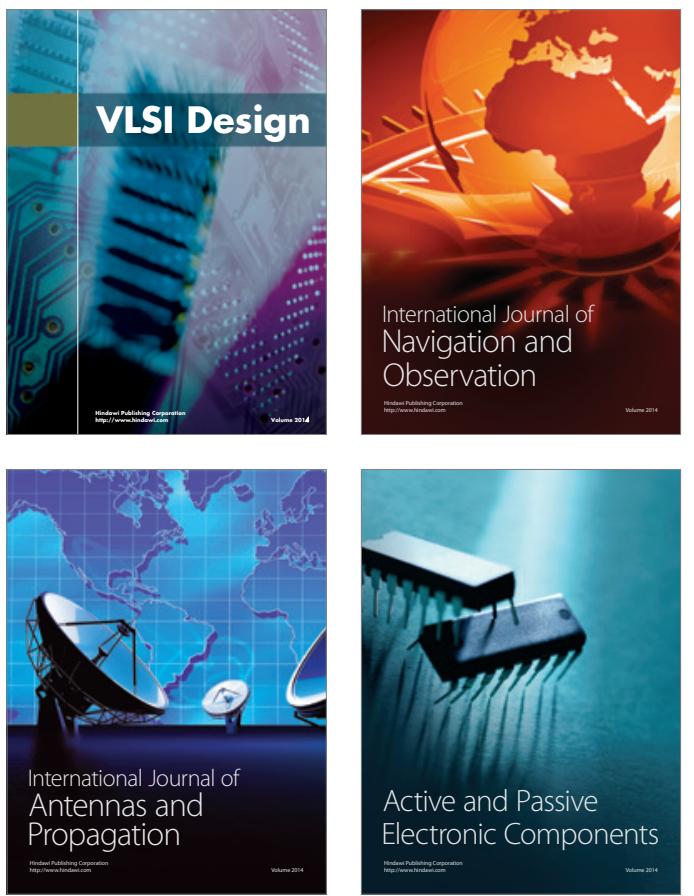
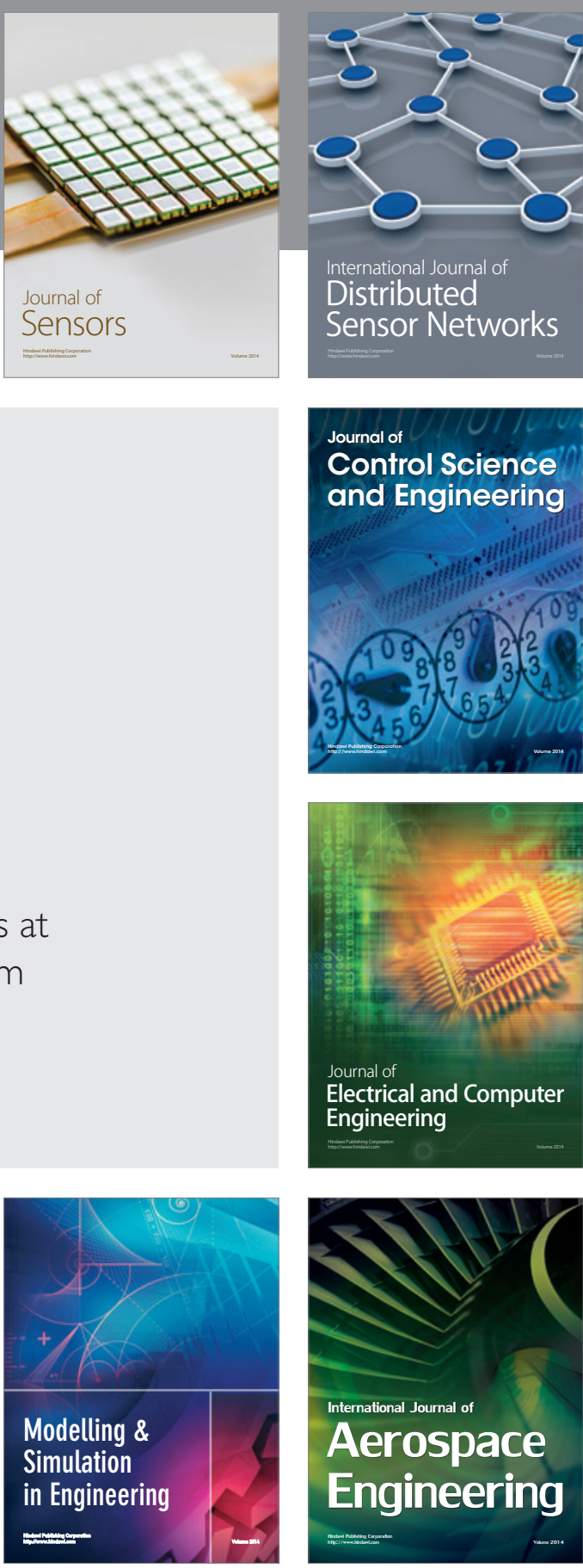

International Journal of

Distributed

Sensor Networks

Journal of

Control Science

and Engineering
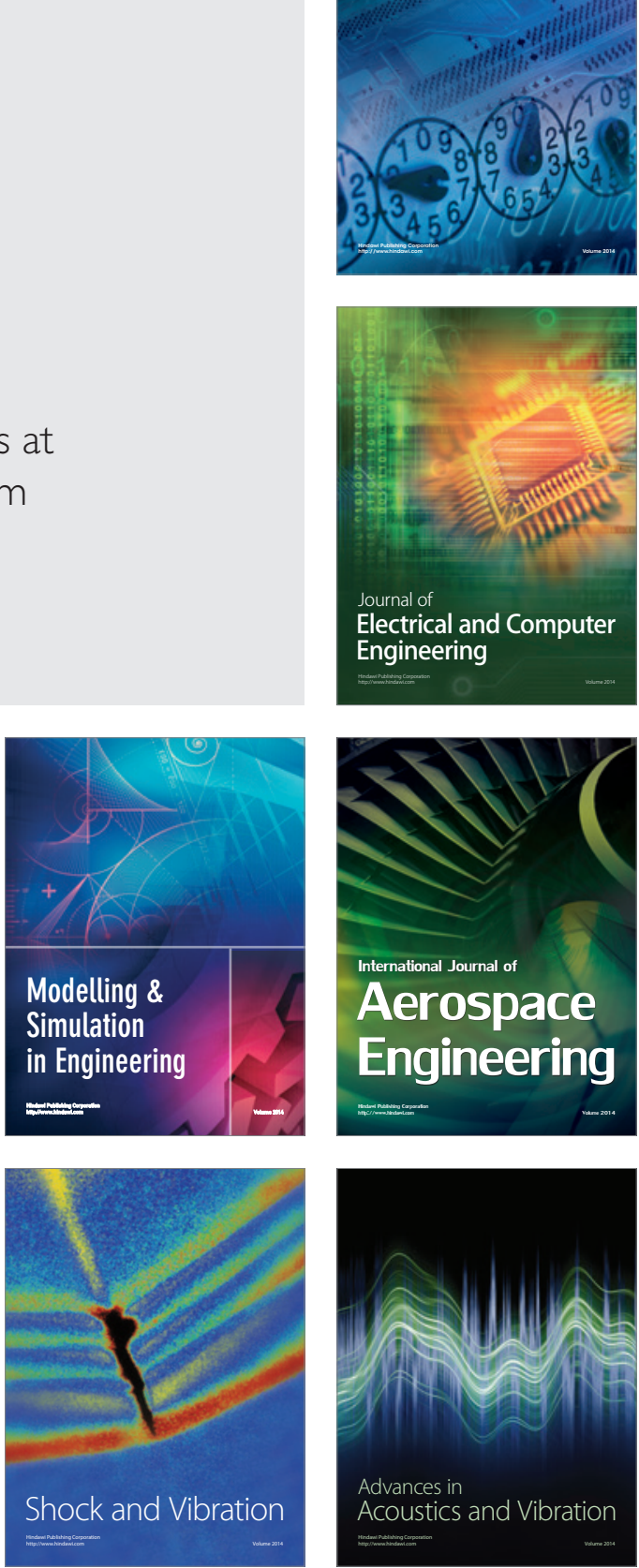